\title{
Take-Off Angle Imaging to Improve Correlation between BSE images and X-ray Maps and to Facilitate Mapping of Samples with Macro-scale Topography
}

\author{
N. C. Barbi ${ }^{1}$ and A. Muto ${ }^{2}$ \\ 1. PulseTor LLC, Pennington, NJ 08534 USA \\ 2. Hitachi High Technologies America, Gaithersburg MD 20878 USA
}

Silicon Photomultipliers (SiPMs) were developed in the 1990s as a solid state alternative to PhotoMultiplier Tubes (PMTs) [1]. More recently, substantial improvement in the technology has been driven by the effort to combine MRI and PET scanners in a single medical imaging instrument, to take advantage of the insensitivity of SiPMs to magnetic fields [2]. Barbi, et.al demonstrated the application to electron microscopes by coupling scintillators directly to SiPMs to create small, efficient, low voltage electron imaging devices [3]. The coupled pair, called a SoM, for Scintillator on Multiplier, eliminates the need for the light pipe and PMT of traditional Everhart Thornley type electron detectors. The small size and simplicity of the SoM devices enable, for example, the mounting of a small PCB comprising two SoMs, each $3 \times 3 \mathrm{~mm}^{2}$, within the electron trap of the SDD (thus creating an electron detector that is integrated within the SDD itself) [4]. The result is a Backscattered Electron Detector (BSED) and Silicon Drift X-ray Detector (SDD) having co-linear axes. The imaging mode associated with the SDDmounted BSED is called Take-Off Angle (TOA) imaging because the electron image is collected from the perspective of the TOA of the SDD.

Figure 1 shows electron and X-ray images of a Mo-containing ferro-silicon material having a rough surface, similar to a fracture surface, mounted on a flat, untilted stub. All SEM images were acquired at $25 \mathrm{kV}$ on an Hitachi S3400N SEM using a Quartz XOne EDS and Image acquisition system equipped with a PulseTor ON-X SDD. Figure 1a is the BSED image obtained using a state-of-the-art annular 4segment solid-state detector in which the segments are arranged under the pole piece of the SEM; Figure $1 \mathrm{~b}$ is the corresponding Mo X-ray Map; Figure 1c is the TOA image. The bright regions within the black rectangle of the BSED image (Figure 1a) are shown to be Mo-rich in the X-ray map (Figure 1b). Based on this correlation, one would also expect that the circled region in the annular BSED image would similarly show high Mo content. It does not. The reason for the absence of this feature in the map is revealed in the TOA image, Figure 1c, which shows that the circled region is topographically shadowed from the X-ray detector.

The correlation between the TOA image and X-ray map demonstrated in Figure 1 suggests that the TOA image can be used to help orient samples with macroscopic topography such that the SDD has a direct view of the region of interest.

Figure 2a shows an optical image of a printed circuit board containing several devices of varying thicknesses, in which the region of interest is defined by the ellipse. The right corner of this region contains a defect. Figure $2 \mathrm{~b}$ is an SEM TOA image showing the same region. In this image, it can be seen that the line of sight to the defect area is shadowed by a neighboring device, with the area of the shadow being highlighted by the yellow box. Figure $2 \mathrm{C}$ is the TOA image after tilting and rotating the sample to achieve an orientation that eliminates the problematic shadowing. Figure $2 \mathrm{~d}$ is the X-ray map for $\mathrm{Sn}$ and $\mathrm{Cu}$, showing that the $\mathrm{Sn}$ has been removed in the defect area, exposing the $\mathrm{Cu}$ underneath [5]. 


\section{References}

[1] B. Dolgoshein,et al, Nuclear Instruments and Methods, Physics Research A 563 (2006) 368-376

[2] C. Piemonte et al, IEEE Transactions on Nuclear Science, 54, No. 1, February (2007) 236-244

[3] N.C. Barbi, et.al. Microsc. and Microanal., 2011

[4] N.C. Barbi, et.al. Microsc. and Microanal., 2012

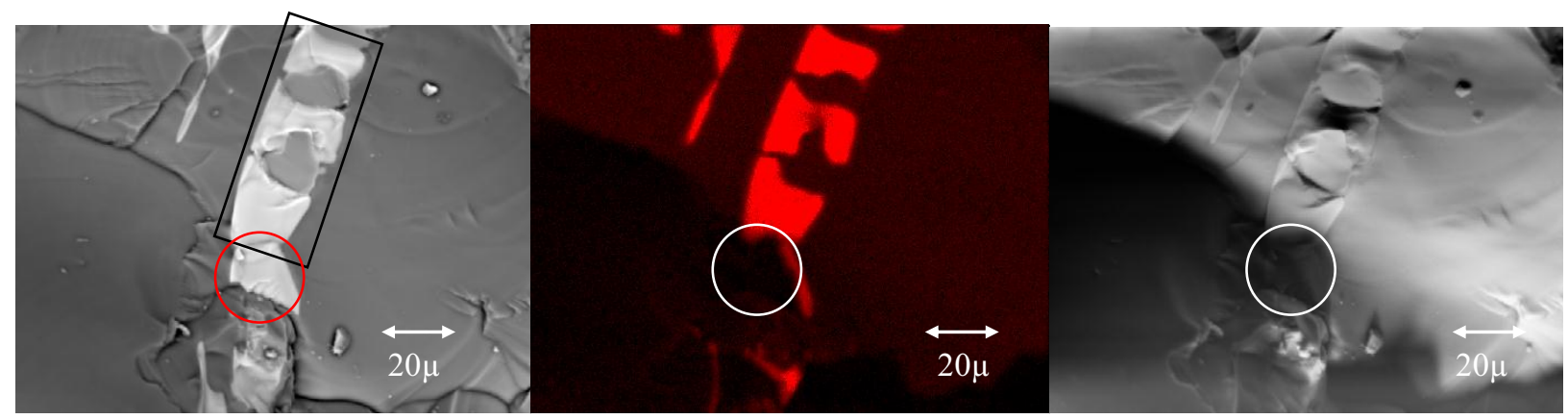

Figure 1a: Annular BSED image Figure 1b: Mo X-ray Map $\quad$ Figure 1c: TOA Image

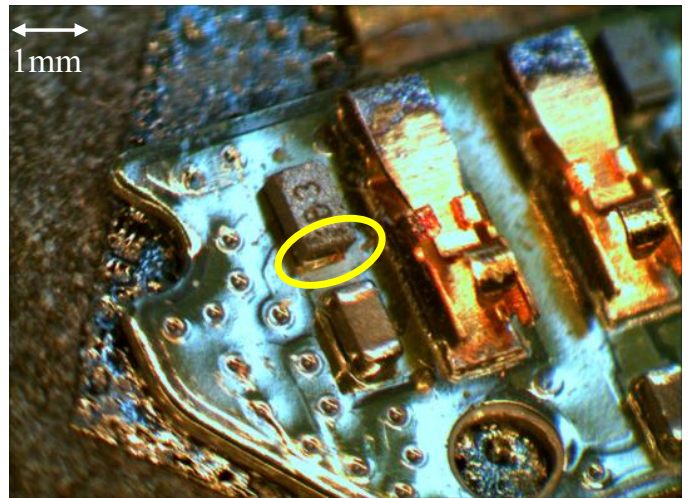

Figure 2a: Stereo-optical image of a populated printed circuit board showing defect region

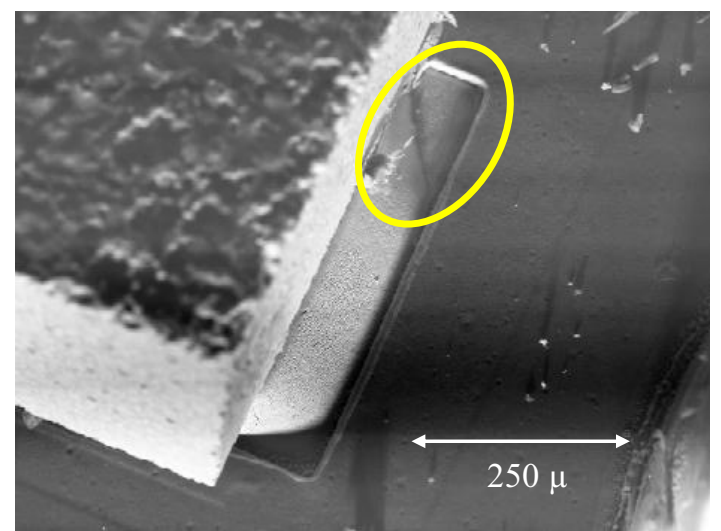

Figure 2c: TOA image after reorientation to eliminate the problematic shadowing

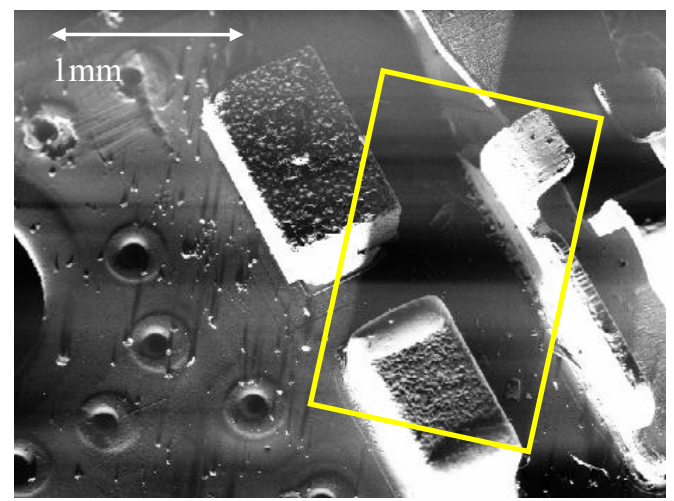

Figure 2b: TOA image showing that the region of interest is shadowed from the SDD

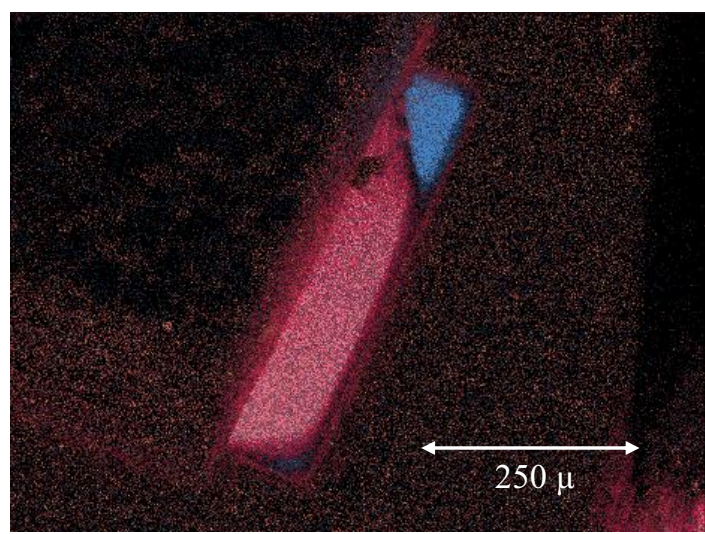

Figure 2d: X-ray map showing that the Sn (pink) has peeled away to reveal the underlying $\mathrm{Cu}$ (blue) 